Open Access

\title{
Maternal vitamin D supplementation during pregnancy and lactation to promote infant growth in Dhaka, Bangladesh (MDIG trial): study protocol for a randomized controlled trial
}

Daniel E. Roth ${ }^{1,2^{*}}$, Alison D. Gernand ${ }^{3}$, Shaun K. Morris ${ }^{1,2}$, Brendon Pezzack ${ }^{1,2}$, M. Munirul Islam ${ }^{4}$, Michelle C. Dimitris ${ }^{1,2}$, Shaila S. Shanta ${ }^{4}$, Stanley H. Zlotkin ${ }^{1,2}$, Andrew R. Willan², Tahmeed Ahmed ${ }^{4}$, Prakesh S. Shah ${ }^{1,5}$, Kellie E. Murphy ${ }^{6}$, Rosanna Weksberg ${ }^{1,7}$, Sanaa Choufani ${ }^{7}$, Rashed Shah ${ }^{8}$ and Abdullah Al Mahmud ${ }^{9}$

\begin{abstract}
Background: Vitamin D regulates bone mineral metabolism and skeletal development. Some observational studies have suggested that prenatal vitamin D deficiency increases the risk of adverse pregnancy and/or birth outcomes; however, there is scant evidence from controlled trials, leading the World Health Organization to advise against routine vitamin D supplementation in pregnancy. Importantly, little is known about the effect of maternal vitamin D status on infant linear growth in communities in South Asia where stunting is highly prevalent and maternal-infant vitamin D status is commonly suboptimal.

Methods/Design: The Maternal Vitamin D for Infant Growth study is a randomized, placebo-controlled, dose-ranging trial of maternal vitamin D supplementation during pregnancy and lactation in Dhaka, Bangladesh. The primary aims are to estimate (1) the effect of maternal prenatal oral vitamin $D_{3}$ supplementation (4200 IU/wk, 16,800 IU/Wk, or 28,000 $\mathrm{IU} / \mathrm{wk}$, administered as weekly doses) versus placebo on infant length at 1 year of age and (2) the effect of maternal postpartum oral vitamin $\mathrm{D}_{3}$ supplementation $(28,000 \mathrm{IU} / \mathrm{wk}$ ) versus placebo on length at 1 year of age among infants born to women who received vitamin D $28,000 \mathrm{IU} / \mathrm{wk}$ during pregnancy. Generally healthy pregnant women $(n=1300)$ in the second trimester $(17-24$ weeks of gestation) are randomized to one of five parallel arms: placebo $4200 \mathrm{IU} / \mathrm{wk}, 16,800 \mathrm{IU} / \mathrm{wk}$, or $28,000 \mathrm{IU} / \mathrm{wk}$ in the prenatal period and placebo in the postpartum period or $28,000 \mathrm{IU} / \mathrm{Wk}$ in the prenatal period and $28,000 \mathrm{IU} / \mathrm{Wk}$ in the postpartum period. Household- and clinic-based follow-up of mother-infant pairs is conducted weekly by trained personnel until 26 weeks postpartum and every 3 months thereafter. The primary trial outcome measure is length for age z-score at 1 year of age. Anthropometric measurements, clinical information, and biological specimens collected at scheduled intervals will enable the assessment of a range of maternal, perinatal, and infant outcomes.

Discussion: The role of vitamin $D$ in maternal and infant health remains unresolved. This trial is expected to contribute unique insights into the effects of improving maternal-infant vitamin D status in a low-income setting where stunting and adverse perinatal outcomes represent significant public health burdens.
\end{abstract}

Trial registration: ClinicalTrials.gov identifier: NCT01924013. Registered on 13 August 2013

Keywords: Bangladesh, Lactation, Length, Linear growth, Nutrition, Pregnancy, Stunting, Vitamin D

\footnotetext{
* Correspondence: daniel.roth@sickkids.ca

'Department of Paediatrics, University of Toronto and the Centre for Global

Child Health, Hospital for Sick Children, 686 Bay Street, Toronto, ON, Canada

${ }^{2}$ Child Health Evaluative Sciences, SickKids Research Institute, Hospital for Sick

Children, 686 Bay Street, Toronto, ON, Canada

Full list of author information is available at the end of the article
} 


\section{Background}

Many low-income countries in Asia have experienced substantial recent reductions in mortality rates among children younger than 5 years of age [1]; for example, Bangladesh has met United Nations Millennium Development Goal 4-a two-thirds reduction in child mortality between 1990 and 2015. However, childhood undernutrition remains a persistent global public health problem [2], particularly in Sub-Saharan Africa and South Asia [3]. In Bangladesh, stunting (height or length less than 2 standard deviations below the standard median for age and sex) was estimated to affect $51 \%$ of children younger than 5 years of age in 2004, but declined to $43 \%$ in 2007 and to $41 \%$ in 2011 according to national surveys [4].

Fetal and early childhood growth is believed to have a critical influence on early childhood infectious disease susceptibility, mortality, and long-term functional and social outcomes, particularly during the first 1000 days of pre- and postnatal development $[5,6]$. Postnatal linear growth faltering - an abnormally slow velocity of length/ height gain that may eventually lead to stunting-often begins early in infancy in low-income settings (i.e., within the first 3 months of life [7]), suggesting an important contribution by prenatal determinants, including epigenetic and endocrine factors that regulate growth in the first months of life. However, the causal pathways implicated in early childhood stunting remain poorly understood $[8,9]$.

Vitamin D is a well-established regulator of bone mineral metabolism and skeletal development [10]. Children with severe vitamin $\mathrm{D}$ deficiency rickets have secondary hyperparathyroidism and demineralization of the growing skeleton, as well as impairment of bone elongation [11]. Vitamin D treatment of affected children leads to an acceleration of linear growth accompanied by resolution of the hyperparathyroidism [11-13]. Effects of improvements in vitamin D status on linear growth in infants and children without rickets have not been widely studied; however, in one trial in India, weekly vitamin D supplementation of low birth weight infants reduced the risk of stunting in adjusted analyses [14]. Vitamin D status is therefore a logical candidate as a modifiable nutritional determinant of linear growth in communities where stunting is prevalent and vitamin D status is commonly suboptimal. In South Asia, there is a high prevalence of biochemical vitamin D deficiency among women and young infants [15]; in Dhaka, we found that $34 \%$ of pregnant women at 26-29 weeks gestation $(N=160)$ had serum 25-hydroxyvitamin D (25(OH)D) concentrations less than $30 \mathrm{nmol} / \mathrm{L}$, and that $64 \%$ had 25(OH)D levels less than $50 \mathrm{nmol} / \mathrm{L}$ [16], a threshold for sufficiency adopted by the Institute of Medicine (IOM) [17]. In the absence of maternal vitamin D supplementation, postnatal infant $25(\mathrm{OH}) \mathrm{D}$ concentration was low in the neonatal period, but gradually increased over the first several months of life (unpublished observations). Therefore, in Bangladesh, we have observed that the period of greatest vulnerability with respect to perinatal vitamin D deficiency overlaps with a critical period of fetal and early infant growth. Because maternal vitamin D status is the predominant determinant of fetal and neonatal vitamin $\mathrm{D}$ stores [18], prenatal vitamin D supplementation represents the optimal approach to testing the effect of improving maternal-infant vitamin D status on fetal-infant growth and other health outcomes.

Observational studies of the association between maternal prenatal vitamin D status and infant anthropometry have yielded conflicting findings [19-23]. The effects of prenatal vitamin D supplementation on infant length have not been widely studied in controlled trials. In a study conducted in the 1970s in London, England, prenatal vitamin D supplementation reduced the risk of small for gestational age (SGA) versus control, but there was no significant effect on birth length [24]; however, the infants in the vitamin D group had significantly greater mean length at 1 year of age [25]. We recently completed a randomized, placebo-controlled, doubleblinded trial of maternal third-trimester vitamin $\mathrm{D}_{3}$ supplementation in Dhaka, in which 160 women were randomized to receive either $35,000 \mathrm{IU} / \mathrm{wk}$ or placebo until delivery. Maternal mean 25(OH)D was significantly higher at delivery after receiving vitamin $\mathrm{D}$ versus placebo ( 134 vs. $39 \mathrm{nmol} / \mathrm{L} ; P<0.001 ; N=133)$. There was a parallel difference in cord $25(\mathrm{OH}) \mathrm{D}(103 \mathrm{nmol} / \mathrm{L}$ in vitamin $\mathrm{D}$ group vs. $39 \mathrm{nmol} / \mathrm{L}$ in placebo group; $P<$ $0.001 ; N=132$ ) [16]. Analyses of the effect of prenatal vitamin D supplementation on infant length among 134 infants followed up to 1 year of age revealed that infants born to women in the vitamin D group had mean length for age z-scores (LAZs) at 1 year that were significantly greater than infants in the placebo group (mean $=0.44$ z-score units higher; 95\% confidence interval [CI], 0.060.82) [26]. This corresponded to an approximate halving of the prevalence of stunting ( $11 \%$ vs. $21 \%)$, which would constitute a substantial public health benefit. The effect appeared to be attributable to a significantly accelerated increase in mean LAZ from birth to 1 month of age (change of $0.53 \mathrm{z}$-score in vitamin $\mathrm{D}$ versus $0.19 \mathrm{z}$ score in placebo group; $P=0.004$ ) rather than a significant difference in birth length [26]. However, some animal [27] and observational [21, 28] studies have suggested an effect of maternal prenatal vitamin D status on growth of the fetus.

There has been considerable recent interest in the role of vitamin $\mathrm{D}$ in maternal and infant health outcomes in low-income settings [29]. Some observational studies have suggested that antenatal vitamin D deficiency increases the risk of adverse pregnancy and birth outcomes, 
including SGA [30, 31]. However, there is scant evidence from controlled clinical trials [31,32], and the World Health Organization (WHO) recently advised against routine vitamin D supplementation in pregnancy until further data become available [33]. Building on our earlier findings of a beneficial effect of prenatal vitamin D supplementation on infant length in a preliminary trial [26], we are conducting a larger dose-ranging trial of maternal vitamin $D_{3}$ supplementation in Dhaka, Bangladesh. The primary aim is to investigate the effect of maternal vitamin D supplementation on infant length at 1 year of age, but the trial also provides an opportunity to study a range of other potential vitamin D-responsive outcomes.

\section{Methods/Design}

The Maternal Vitamin D for Infant Growth (MDIG) trial is randomized, placebo-controlled, dose-ranging trial of maternal vitamin $\mathrm{D}$ supplementation during pregnancy and lactation to improve infant linear growth in Dhaka, Bangladesh (ClinicalTrials.gov identifier NCT01924013). A detailed trial protocol was developed in accordance with the SPIRIT guidelines [34] through a collaboration between the International Centre for Diarrhoeal Disease Research (ICDDR,B; Dhaka, Bangladesh) and the Hospital for Sick Children (Toronto, ON, Canada) and with funding from the Bill and Melinda Gates Foundation through its Achieving Healthy Growth platform (http:/gcgh.grandchallenges.org/grantopportunities/ pages/healthygrowth.aspx).

The first participant was enrolled on 18 March 2014; enrollment is expected to proceed until August 2015; and planned data collection will continue until 2018.

\section{Objectives}

The primary aims of the trial are to estimate (1) the effect of maternal prenatal oral vitamin $\mathrm{D}_{3}$ supplementation (4200 IU/wk, $16,800 \mathrm{IU} / \mathrm{wk}$, or $28,000 \mathrm{IU} / \mathrm{wk}$ administered as weekly doses) versus placebo on infant length at 1 year of age in Dhaka, Bangladesh; and (2) the effect of maternal postpartum oral vitamin $\mathrm{D}_{3}$ supplementation $(28,000 \mathrm{IU} / \mathrm{wk})$ versus placebo on length at 1 year of age among infants born to women who received vitamin D 28,000 IU/wk during pregnancy. Secondary anthropometric outcomes include birth size and the prevalence of SGA, the prevalence of stunting at 1 year of age, and mean attained LAZ and stunting prevalence at 2 years of age.

Clinical, biochemical, and microbiological surveillance of enrolled mother-infant pairs will enable a range of secondary objectives to be addressed, including (1) estimation of the effect of maternal vitamin D supplementation on the incidence of acute respiratory infections (ARIs) during early infancy; (2) exploration of the roles of specific hormones, nutrients, environmental contaminants, and inflammatory markers in the mediation or modification of the effect of vitamin D on fetal and infant growth, with particular emphasis on the role of the parathyroid hormone (PTH) system; and (3) investigation of the role of epigenetic modification of genes involved in perinatal vitamin D metabolism in infant stunting.

\section{Setting and participants}

Recruitment, enrollment, and clinical activities are based at the Maternal and Child Health Training Institute (MCHTI), a Bangladeshi national governmentoperated facility that provides health care to pregnant women and children in its referral area in central Dhaka, Bangladesh. MCHTI has outpatient antenatal care clinics, a labor and delivery unit, and inpatient pediatric services. The Dhaka wards and/or unions near MCHTI that are included in the trial area include Kamrangirchar, Azimpur, Lalbag, and Hazaribag. About three-fourths of the participants are expected to be residents of Kamrangirchar, a group of urban slums on the Buriganga River along the periphery of Dhaka city. The area is densely populated, with a total population of about 300,000, of whom approximately 265,000 reside in slum settlements [35]. The literacy rate is approximately $29 \%$ (compared with the national average of 32 $\%$ ) and more than $30 \%$ of the residents have monthly incomes of 5000 taka (about US\$60) or less. Income earners are mainly day laborers (e.g., rickshaw pullers), and many men work in local tanneries or operate small businesses. However, the socioeconomic status of residents varies, owing to the presence of some universities and government offices in the area.

The MDIG trial is an efficacy study designed to test a specific biological mechanism implicated in fetal and infant growth. We therefore established a detailed set of eligibility criteria that will optimize the internal validity of the trial by minimizing the number of withdrawals due to loss to follow-up or serious adverse events, acknowledging that this may theoretically reduce the generalizability of the findings. Among women presenting to MCHTI for antenatal care, participant eligibility is based on the following inclusion criteria: age 18 years and older, intention to reside in the trial area for at least 18 months, and currently at 17-24 completed weeks of gestation (i.e., 17 weeks +0 days to 24 weeks +0 days, inclusive) based on recalled last menstrual period (LMP) and ultrasound performed by MCHTI technicians (Just Vision 400, Toshiba, Tokyo, Japan; SonoAce X8, Samsung Medison, South Korea). All potential participants undergo routine ultrasound. If there is a difference of more than 10 days between gestational age (GA) calculated using the recalled LMP and secondtrimester ultrasound, the estimated date of delivery is adjusted as per the second-trimester ultrasound. Otherwise, 
the GA date based on recalled LMP is used [36]. If more than one ultrasound report is available, GA estimation is based on the earliest of the ultrasounds. If the earliest ultrasound was performed in the first trimester and there is a difference of more than 5 days between GA calculated using the recalled LMP and first-trimester ultrasound, the estimated date of delivery is adjusted as per the firsttrimester ultrasound [36].

Pregnant women are not enrolled if any of the following exclusion criteria apply: self-reported history of any medical condition or medications that may predispose to vitamin D sensitivity, altered vitamin D metabolism, and/or hypercalcemia (e.g., active tuberculosis or current therapy for tuberculosis, sarcoidosis, history of renal and/or ureteral stones, parathyroid disease, renal or liver failure, or current use of antiseizure medications); high-risk pregnancy based on point-of-care (POC) testing (hemoglobin $[\mathrm{Hb}]<70 \mathrm{~g} / \mathrm{L}$; proteinuria, defined as $\geq 300 \mathrm{mg} / \mathrm{dl}$ based on urine dipstick; hypertension, defined as systolic blood pressure $\geq 140 \mathrm{mmHg}$ and/or diastolic blood pressure $\geq 90 \mathrm{mmHg}$ ); high-risk pregnancy based on maternal report and/or past ultrasound findings (multiple gestation, major congenital anomaly, severe oligohydramnios); unwillingness to stop taking non-study vitamin D or calcium supplements or a multivitamins containing calcium and/or vitamin D; currently prescribed vitamin $\mathrm{D}$ supplements as part of a physician's treatment plan for vitamin D deficiency; and/or previous enrollment in the MDIG trial during a prior pregnancy.

All participants provide written informed consent before randomization. The informed consent process is undertaken in conjunction with the iterative eligibility assessment: preliminary screening (based on age, location of residence, plans to remain in referral area), detailed medical screening by a study physician, POC testing (blood pressure, urine dip, $\mathrm{Hb}$ ), and obstetric ultrasound. Women may choose to exit the enrollment process at any time. Among prospective participants who complete the screening process and are found to meet all criteria, a detailed discussion of the trial is undertaken before written informed consent is sought. Eligible pregnant women are encouraged to discuss trial participation with their spouse and/or other family members before finalizing consent. Two additional consent procedures are undertaken at a later time for supplemental study procedures: (1) husbands of enrolled participants are approached to obtain consent for paternal anthropometry and blood specimen collection, and (2) enrolled participants are approached at or beyond 30 weeks of gestation to request the participation of their infant in an ARI substudy during the 0- to 6-month postnatal period (detailed methods of the ARI substudy will be presented elsewhere).

\section{Design and sample size}

The MDIG trial is a randomized, placebo-controlled, double-blinded, dose-ranging trial with a 1:1 allocation ratio across five parallel groups (Fig. 1). A total of 1300 pregnant women will be randomized, with a target of allocating 260 women in each group (Fig. 1). The primary outcome analysis will be a between-group comparison of mean LAZs at 1 year of age, assuming up to $15 \%$ attrition from each group before this time point. To assess the effect of prenatal vitamin $\mathrm{D}$, we plan to perform five primary between-group analyses, comprising each vitamin D dose versus placebo (three comparisons) and comparisons between adjacent doses (two comparisons). A conservative approach to addressing multiple testing is to partition the $\alpha$ (risk of type I error) among the five comparisons (conventional overall 0.05 divided by 5); thus, each between-group comparison will be tested as a two-sided test with an $\alpha$ of 0.01 , assuming $90 \%$ power. With 220 analyzable participants per group (about $85 \%$ of total enrolled), the minimum detectable difference in LAZ will be $0.40 \mathrm{z}$-score units, which equates to approximately $1.0 \mathrm{~cm}$ at 1 year of age based on WHO growth standards. In our preliminary trial, we observed an increase in LAZ of 0.44 at 1 year attributable to prenatal vitamin $\mathrm{D}$ supplementation, which corresponded to an increase of $1.1 \mathrm{~cm}(95 \% \mathrm{CI}, 0.06-2.0)$, adjusted for sex [26].

The secondary analysis at 2 years of age will enable detection of an approximately $1.2-\mathrm{cm}$ difference between groups. For the postpartum effect analysis of 28,000 IU/ wk versus placebo postpartum among women who received 28,000 IU/wk antenatally, the comparison at 1 year of age will have a minimum detectable difference of $0.31 \mathrm{z}$-score units (assuming $90 \%$ power, a $5 \%$ risk of a type I error for a two-sided test, and at least 220 participants per group). Based on WHO growth standards, this is approximately $0.77 \mathrm{~cm}$ at 1 year of age and $0.96 \mathrm{~cm}$ at 2 years of age. Because the $\alpha$ is not subject to multiple testing for the postpartum effect analysis, there is greater precision to detect a smaller difference compared with the prenatal effect analysis. In order to enroll 1300 participants, we expect to provisionally screen approximately 15,000 pregnant women for eligibility. The major reasons for exclusion during preliminary screening are GA beyond 24 weeks and residence outside the study area.

\section{Randomization and allocation concealment}

The allocation sequence was generated by the trial statistician (ARW) using a computer-generated random number sequence according to a simple randomization scheme (i.e., no stratification or blocking) [37]. The list was provided to the Toronto Institute for Pharmaceutical Technology (TIPT), the pharmaceutical company 


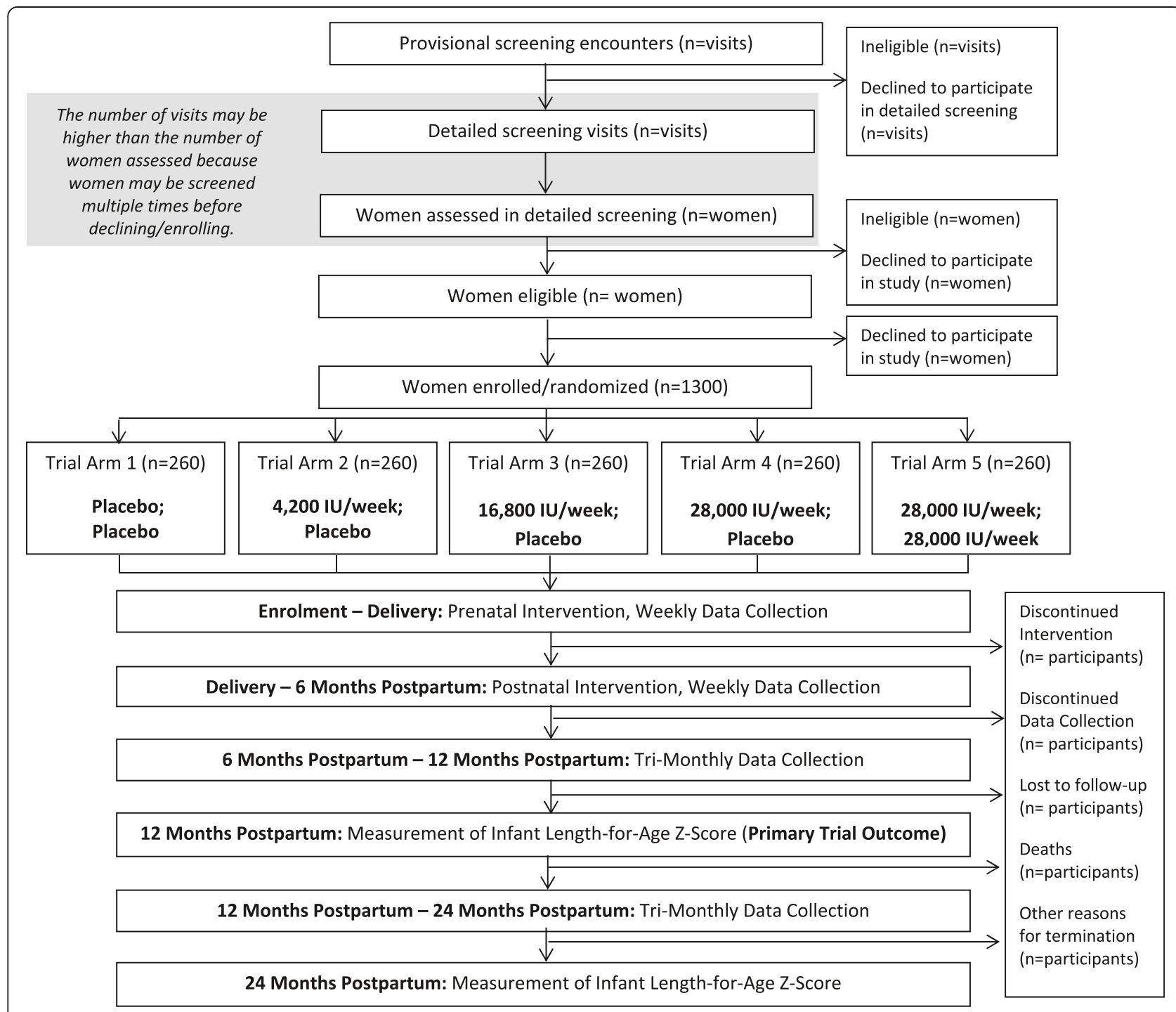

Fig. 1 Maternal Vitamin D for Infant Growth (MDIG) trial flow diagram. The allocated intervention in each group is shown as "prenatal intervention; postpartum intervention"

that has produced and packaged the supplements in individual participant supplement packs, each of which is labeled with a unique participant identifier. The prelabeled supplement packets containing both prenatal and postpartum supplements (in separate vials) are sequentially allocated to participants according to their order of enrollment. Participants, investigators, field personnel, study laboratory staff, and data analysts are blinded to vitamin $\mathrm{D}$ or placebo group allocation. The allocation scheme will be made available to the Data and Safety Monitoring Board (DSMB) in cases in which individual participants need to be unmasked because of suspected supplement-related adverse events (i.e., hypercalcemia or clinical features suggestive of vitamin D toxicity).

\section{Interventions and control}

\section{Vitamin $D$ doses}

The primary objective of the MDIG trial is to establish evidence of a causal relationship between maternal-infant vitamin D status and infant stature. A randomized doseranging trial enables robust causal inferences based on dose-response effects, in addition to relatively unbiased comparisons with the placebo group. Three vitamin $D_{3}$ doses (4200 IU/wk, 16,800 IU/wk, and 28,000 IU/wk) were selected on the basis of preliminary data, published literature, and consideration of the 2010 IOM dietary reference intakes [38] (see Additional file 1 for detailed rationale). Pharmacological principles and empirical data indicate that weekly doses of vitamin D achieve $25(\mathrm{OH}) \mathrm{D}$ 
levels similar to equivalent daily doses [39]; thus, the equivalent daily doses in increasing order are $600 \mathrm{IU} /$ day, $2400 \mathrm{IU} /$ day, and $4000 \mathrm{IU} /$ day. Our previous pharmacokinetic findings among pregnant women in Dhaka $[16,40]$ have generally been consistent with published estimates of the vitamin D-to-25(OH)D dose-response relationship in non-pregnant adults (i.e., an approximately $0.7 \mathrm{nmol} / \mathrm{L}$ increase in $25(\mathrm{OH}) \mathrm{D}$ at steady state for each $1 \mu \mathrm{g} /$ day of vitamin $\left.\mathrm{D}_{3}[41,42]\right)$. The preliminary pharmacokinetic studies have provided evidence of the biochemical efficacy, tolerability, and short-term safety of doses of up to 35,000 $\mathrm{IU} / \mathrm{wk}$ in pregnancy in the target population. Hollis et al. previously reported that doses up to $4000 \mathrm{IU} /$ day were well tolerated and efficacious at raising 25(OH)D in pregnant women in the United States [43].

\section{Study tablets}

Oral vitamin $\mathrm{D}_{3}$ (cholecalciferol) is provided as small tablets (10 $\mathrm{mm}$ diameter) that are custom-manufactured by TIPT in Toronto, ON, Canada (www.tipt.com). Each participant's weekly dose consists of a single tablet. Across trial groups, the tablets vary only by the vitamin $\mathrm{D}_{3}$ dose but are identical with respect to appearance, taste and excipients (Table 1). Vitamin $\mathrm{D}_{3}$ content per tablet has been verified in each production batch (Table 2), with a very high degree of tablet uniformity (F. Martinuzzi, personal communication, December 2014).

Table 1 Vitamin $D_{3}$ and excipient content of custom-made tablets for the Maternal Vitamin D for Infant Growth trial ${ }^{a}$

\begin{tabular}{ll}
\hline Content type & Tablet contents \\
\hline Granules $^{\mathrm{b}}$ & ${\text { Vitamin } \mathrm{D}_{3}^{c}}^{\mathrm{a}}$ \\
& a-Tocopherol $^{\mathrm{d}}$ \\
& Corn starch \\
& Medium-chain triglycerides \\
& Acacia gum \\
& Sucrose \\
& Dibasic calcium phosphate \\
Additional tablet excipients & Croscarmellose sodium \\
Tablet coating & Silicon dioxide \\
& Hydroxypropylmethylcellulose \\
& Polyethylene glycol 6000 \\
& Titanium dioxide \\
\hline
\end{tabular}

${ }^{a}$ Composition information provided by the manufacturer (Toronto Institute of Pharmaceutical Technology, Toronto, ON, Canada)

${ }^{b}$ Placebo granules were identical except for the absence of vitamin $D_{3}$ 'Dry vitamin $D_{3} 100$ GFP/HP (BASF, Mississauga, ON, Canada)

${ }^{d} a$-Tocopherol is included as an antioxidant to stabilize the vitamin $D_{3}$. The vitamin E content of each tablet is $2.8 \mathrm{mg} / \mathrm{wk}$ (about $0.4 \mathrm{mg} /$ day), which is far below recommended dietary allowances for pregnancy ( $15 \mathrm{mg} /$ day) and lactation (19 mg/day)

\section{Supplement administration}

Supplementation begins at enrollment (second trimester) and continues on a weekly basis throughout pregnancy and the postpartum period until 26 weeks postpartum (6 months). Study personnel maintain and store all tablet supplies in locked study offices and directly observe tablet ingestion during home or clinic visits. However, for periods of up to 4 weeks during which the participant plans to travel or during holidays, advance doses may be given to the participant to take unobserved but with telephone reminders. Tablets are swallowed whole with any liquid (e.g., water, juice, tea) or, if necessary, chewed with or without a small amount of soft food. Tablets may be consumed with or without food, which has a negligible impact on vitamin $\mathrm{D}$ absorption [44]. Although scheduled for specific 7-day intervals, a missed dose may be administered as a late dose on any day up to 7 days after the scheduled date of administration; thereafter, subsequent dosing continues as originally scheduled, even if this incurs at an interval of less than 7 days between the late dose and the subsequent regularly scheduled dose. If a participant refuses a dose or complains of feeling unwell (e.g., nausea) on the scheduled day of dose administration, the dose may be deferred for up to 7 days. If a participant vomits during or within 20 minutes of dose administration (observed by study personnel), the dose may be repeated immediately or may be deferred to another day (within 7 days). If vomiting occurs more than 20 minutes after a dose was swallowed, the dose is considered successfully administered. If a participant refuses doses for 3 consecutive weeks and in the third week expresses the intention to continue avoidance and/or refusal of the dose, weekly supplementation may be discontinued, but clinical follow-up and scheduled data collection procedures may continue.

\section{Micronutrient cointerventions}

Calcium 500 mg/day as calcium carbonate (Calbo; Square Pharmaceuticals, Dhaka, Bangladesh) and iron and folic acid (66 mg elemental iron per day, and $350 \mu \mathrm{g}$ folic acid per day included in the standard formulation available in Bangladesh) are provided to all participants throughout the intervention phase (prenatal period and up to 6 months postpartum). Although prenatal calcium supplementation is not formally recommended or widely practiced in Bangladesh, standard provision of calcium was incorporated into the trial design to mitigate any ratelimiting effects of serious maternal dietary calcium deficits on fetal growth or other outcomes. From a practical standpoint, the investigation of vitamin D supplementation in the context of routine calcium supplementation will facilitate the translation of trial findings into anticipated future contexts in which supplemental calcium is 
Table 2 Vitamin $D_{3}$ content of custom-made trial tablets, based on postproduction coated tablet content analysis by highperformance liquid chromatography ${ }^{a}$

\begin{tabular}{|c|c|c|c|}
\hline \multirow{2}{*}{$\begin{array}{l}\text { Label } \\
\text { claim }\end{array}$} & \multicolumn{3}{|c|}{ Cholecalciferol content (\% label claim) } \\
\hline & $\begin{array}{l}\text { Batch } 1^{\mathrm{b}} \\
\text { (November 2013) }\end{array}$ & $\begin{array}{l}\text { Batch } 2 \\
\text { (April 2014) }\end{array}$ & $\begin{array}{l}\text { Batch } 3 \\
\text { (June 2014 }\end{array}$ \\
\hline $4200 \mathrm{IU}$ & $106 \%$ & $103 \%$ & $101 \%$ \\
\hline $16,800 \mathrm{IU}$ & $105 \%$ & $101 \%$ & $102 \%$ \\
\hline $28,000 \mathrm{IU}$ & $102 \%$ & $101 \%$ & $99 \%$ \\
\hline
\end{tabular}

${ }^{a}$ Modified United States Pharmacopeia method, as reported by the manufacturer (Toronto Institute of Pharmaceutical Technology, Toronto, ON, Canada). Results shown reflect the batches completed and assayed at the time of manuscript preparation, not the full set of batches to be used in the trial. ${ }^{b}$ Similar results obtained in testing by independent third-party laboratory (Alpha Laboratories, Toronto, ON, Canada)

more routinely provided. We considered the recent recommendation by the WHO advising 1.5-2 g/day of supplemental calcium for pregnant women with low dietary calcium intake [45], but this policy has not been widely adopted, owing to substantial logistical and technical limitations. Supplemental calcium and/or vitamin D not prescribed by the study protocol are prohibited during the intervention phase of the trial. Participants are questioned on a weekly basis as to whether they have consumed other nutrient supplements. Participants who continue to consume non-study supplements after an initial warning are no longer permitted to continue receiving the allocated intervention, although clinical follow-up and scheduled data collection procedures may continue.

\section{Visit schedule}

Following enrollment and randomization of pregnant women at 17-24 weeks of gestation, weekly supplementation (vitamin D or placebo) occurs throughout the prenatal period and during the first 6 months postpartum. Data collection continues during the non-intervention phase beginning at 26 weeks postpartum. The primary anthropometric outcome will be ascertained at 1 year of age, and follow-up of infants will continue until 2 years of age. The prenatal timeline of study activities is structured in terms of GA (in weeks), based on the recalled LMP and/or ultrasound results. Postnatal follow-up visits are scheduled on the basis of the infant's age in weeks (rather than anniversaries of the birthdate); however, the timing of major visits is operationally described in terms of months or years for simplicity (e.g., 3 months $=13$ weeks, 6 months $=26$ weeks, 9 months $=39$ weeks, 1 year $=12$ months $=52$ weeks, and 2 years $=24$ months $=104$ weeks).

Participant visits are scheduled on a weekly basis during the prenatal and 6-month postpartum intervention phase. The majority of visits are conducted in the participants' homes; however, selected visits that involve specimen collection or anthropometry are preferentially conducted in a clinic at MCHTI. Participants are strongly encouraged to seek a facility-based delivery at MCHTI; however, perinatal data and specimen collection are attempted when feasible for home deliveries or for deliveries that occur at facilities other than MCHTI. Beyond 6 months, clinic-based visits occur at 3-month intervals until 2 years of age (Additional file 2). Participant retention is promoted through frequent interaction between field-level study personnel and the participants (weekly during the intervention phase). Adherence to the schedule of clinic visits is facilitated by compensation of participants for costs of transportation and time away from the home and/or work.

All study activities (e.g., supplementation, visits, maternal and infant specimen collection) cease for a motherinfant pair when any one of the following events occurs: (1) the 24-month infant visit is completed, (2) participant (maternal) death before delivery, (3) stillbirth or infant/child death, (4) consent for all types of follow-up is withdrawn, or (5) loss to follow-up. Loss to follow-up is considered to have occurred if (1) study staff determine conclusively that the participant cannot be contacted for the purposes of data collection for the duration of the period of scheduled follow-up (e.g., they have been informed of the participant's emigration from Bangladesh) or (2) at least 3 months have passed since the scheduled but missed 24-month postnatal visit. A participant may be absent for a period of time (and thus miss supplement dosing, specimen collection, and other data collection) and yet return to follow-up without being excluded from the study.

\section{Data collection procedures}

Trained personnel collect participant data using questionnaires, POC clinical tests, abstraction of prenatal ultrasound reports, anthropometric measurements, and specimen collection throughout the interventional and observational phases of the study (Additional file 2). Data are recorded using a series of standardized data collection forms that are customized for each visit type. To overcome low literacy in the population, field personnel administer all questionnaires as structured oral interviews. A wide range of lifestyle and health-related items (e.g., obstetric history, tobacco use, sun exposure) are included in baseline and follow-up questionnaires. Dietary intake of vitamin $\mathrm{D}$, calcium, phosphorus, and phytates are assessed among participants (mothers) at enrollment and 6 months postpartum using a customized, focused food frequency questionnaire. Infant dietary patterns are assessed at weekly visits to assess breastfeeding patterns and timing of introduction and frequency of consumption of specific complementary foods. A household survey to assess socioeconomic status is conducted at the first scheduled home 
visit 1 week after enrollment, at the 9-month home postnatal visit, and at the 21-month postnatal home visit.

POC tests include blood pressure measurement, urine dipstick testing, and $\mathrm{Hb}$ concentration estimation. Maternal systolic and diastolic blood pressure is measured using an automated digital blood pressure monitor (WatchBP Home; MicroLife USA, Clearwater, FL, USA). Two readings are taken at least 1 minute apart and recorded; if either diastolic or systolic measurements differ between the paired readings by more than $10 \mathrm{mmHg}$, a third measurement will be performed. For analysis, paired readings will be averaged; where a third reading has been obtained, the single discordant reading will be excluded. Urine dipsticks (Urinalysis Reagent Test Strips; Siemens Diagnostics, Erlangen, Germany) are used at enrollment as part of the eligibility assessment and then after enrollment if high blood pressure is detected (systolic blood pressure $\geq 140 \mathrm{mmHg}$ and/or diastolic blood pressure $\geq 90 \mathrm{mmHg}$ ) or if there are symptoms (e.g., dysuria) suggestive of urinary tract infection (to test for leukocyte esterase). Maternal $\mathrm{Hb}$ will be measured at enrollment (as part of the eligibility screening process) in a finger-stick blood sample using a handheld hemoglobinometer (Hb 201; HemoCue, Ängelholm, Sweden). Infant $\mathrm{Hb}$ is measured using the same HemoCue device at 6 months of age.

Standardized procedures for infant length, weight, and head circumference $(\mathrm{HC})$ measurements were adapted from the INTERGROWTH-21st study standard operating procedures [46]. Other published resources were adapted to develop standard protocols for measurement of upper arm length (UAL) [47], mid-upper arm circumference (MUAC) [48], and rump-to-knee length (RKL) [49]. Infants are measured independently by two study personnel at each visit. The paired measurements are compared, and if they differ by more than the threshold values $(7 \mathrm{~mm}$ for length; $5 \mathrm{~mm}$ for $\mathrm{HC}$, UAL, MUAC, and RKL; and $50 \mathrm{~g}$ for weight), a second set of measurements is performed. If the second pair of measurements differs by more than the threshold values, the procedure is repeated a third time (except for infant weight, which is measured using a digital scale and thus expected to be concordant). Length, weight, and $\mathrm{HC}$ are measured at birth, at one randomly assigned weekly visit during the first 2 months after birth, and then every 3 months starting at the 3 -month visit. MUAC, UAL, and RKL are measured at 3, 6, 12, and 24 months; UAL and RKL are also measured at birth.

Length, HC, MUAC, UAL, and RKL are recorded to the last completed unit. For each infant at each time point, the mean of acceptable paired measures will be used in primary analyses. Infant weight is measured to the nearest $5 \mathrm{~g}$ (up to $10 \mathrm{~kg}$ ) and to the nearest $10 \mathrm{~g}$ (for $>10 \mathrm{~kg}$ ) using a digital infant scale (seca 334; Seca,
Hamburg, Germany). Length is measured to the last completed $0.1 \mathrm{~cm}(1 \mathrm{~mm})$ using a wooden length board (Infant/Child ShorrBoard; Weigh and Measure, Olney, MD, USA). Initially, we selected a length board with a counter display and a ball bearing mounted sliding footboard (Harpenden infantometer; Holtain, Crymych, UK), but decalibration of the counter occurred frequently, particularly when the instrument was moved among assessment locations. Therefore, as of 15 December 2014, the ShorrBoard was adopted because of its easier portability and robustness. HC, MUAC, and UAL are measured using a flexible tape measure, and RKL is measured using a rigid caliper (Holtain-Kahn Abdominal Caliper; Holtain). These dimensions are measured to the last completed $0.1 \mathrm{~cm}(1 \mathrm{~mm})$. An anthropometric standardization workshop was conducted before launch of anthropometric data collection, which was adapted from the methods of the WHO [50] and the INTERGROWTH-21st study group [51].

Maternal and paternal weight and height are measured at MCHTI according to standard methods adapted from the National Health and Nutrition Examination Survey Anthropometry Procedures Manual of the Centers for Disease Control and Prevention [47], using a digital floor scale (HD-318; Tanita, Tokyo, Japan) and a stadiometer (Leicester Height Measure device; Chasmors, London, UK). Measurements are performed in duplicate. Third measurements are taken if the following discrepancies are noted between the paired measures: more than 0.5 $\mathrm{kg}$ for weight and more than $2 \mathrm{~cm}$ for height.

\section{Specimen collection}

\section{Blood specimen collection}

Trained phlebotomists collect maternal blood, paternal blood, cord venous and arterial blood, and infant blood specimens according to standard sampling procedures (Table 3). Following filling, BD Vacutainer serum (red top) and ethylenediaminetetraacetic acid (EDTA) (lavender or blue top) blood collection tubes (BD Diagnostics, Franklin Lakes, NJ, USA) are inverted according to the manufacturer's instructions and centrifuged immediately (EDTA) or after 30 minutes (serum) at low speed for 15 minutes, and the supernatant (plasma or serum) is transferred via micropipettes in $0.25-\mathrm{ml}$ aliquots into prelabeled microfuge tubes (Fisherbrand Free-Standing Microcentrifuge Tubes with Screw Caps; Thermo Fisher Scientific, Waltham, MA, USA). Whole blood aliquots (1.5 or $0.5 \mathrm{ml}$, depending on size of blood draw and laboratory requirements) are drawn from EDTA tubes immediately after mixing (before centrifugation) for paternal blood, maternal blood at delivery, and cord venous blood specimens. Within 30 minutes of blood collection, serum and plasma aliquots are placed in a portable ultracold freezer (Shuttle ULT-25N; Stirling Ultracold, Athens, OH, 
Table 3 Biological specimen collection schedule and planned laboratory analyses in the Maternal Vitamin D for Infant Growth (MDIG) trial in Dhaka, Bangladesh

\begin{tabular}{|c|c|c|c|c|c|c|c|c|c|c|c|}
\hline & & \multicolumn{6}{|c|}{ Maternal-fetal } & \multicolumn{3}{|c|}{ Infant } & \multirow[t]{2}{*}{ Father } \\
\hline & & Baseline & $\begin{array}{l}30 w k \\
\text { gestation }\end{array}$ & Delivery & $\begin{array}{l}\text { Cord } \\
\text { blood }\end{array}$ & $\begin{array}{l}3 \text { mo } \\
\text { postpartum }\end{array}$ & $\begin{array}{l}6 \text { mo } \\
\text { postpartum }\end{array}$ & $\begin{array}{l}3 \\
\mathrm{mo}\end{array}$ & $\begin{array}{l}6 \\
\mathrm{mo}\end{array}$ & $\begin{array}{l}12 \text { and } \\
24 \mathrm{mo}\end{array}$ & \\
\hline \multirow{11}{*}{$\begin{array}{l}\text { Blood/serum/ } \\
\text { plasma }\end{array}$} & Calcium & $\mathrm{R}^{\mathrm{a}}$ & $R$ & $R$ & $R$ & $R$ & $R$ & $R$ & $R$ & & \\
\hline & $\begin{array}{l}\text { Phosphate, alkaline phosphatase, } \\
\text { creatinine }\end{array}$ & & & & & & & & $S^{a}$ & & \\
\hline & $25(\mathrm{OH}) \mathrm{D}$ & $\mathrm{R}$ & & S & $\mathrm{S}$ & & S & S & S & & \\
\hline & $\begin{array}{l}\text { 1,25(OH) })_{2} \mathrm{D} \text {, whole/bioactive PTH(1-84), } \\
\text { C-terminal PTH(73-84), intact PTH, FGF-23 }\end{array}$ & S & & S & S & & & S & S & & \\
\hline & PTHrP & S & & S & $\mathrm{S}$ & S & S & S & S & & \\
\hline & IGF-1, IGFBP-1, IGFBP-3 & & & & $S$ & & & & S & & \\
\hline & Ferritin & & & & & & & & S & & \\
\hline & Retinol, folate, cadmium & & & S & & & & & & & \\
\hline & C-reactive protein, IL-6, IL-8, TNF-a & & & $S$ & $S$ & & & & & & \\
\hline & Epigenetic studies & & & S & $S$ & & & & & & S \\
\hline & Collection for future analyses & $\mathrm{R}$ & R & $\mathrm{R}$ & $\mathrm{R}$ & $\mathrm{R}$ & $\mathrm{R}$ & $\mathrm{R}$ & $\mathrm{R}$ & $\mathrm{R}$ & $\mathrm{R}$ \\
\hline Placenta & Epigenetic studies & & & $\mathrm{R}$ & & & & & & & \\
\hline \multirow[t]{3}{*}{ Urine } & Calcium:creatinine ratio & & & $\mathrm{R}$ & & & & & S & & \\
\hline & Calcium, phosphate, fluoride, cyclic AMP & & & & & & & & S & & \\
\hline & Collection for future analyses & $\mathrm{R}$ & & $\mathrm{R}$ & & & & & $\mathrm{R}$ & & \\
\hline \multirow[t]{2}{*}{ Breast milk } & PTHrP & & & & & S & & S & & & \\
\hline & Collection for future analyses & & & & & $\mathrm{R}$ & & $\mathrm{R}$ & & & \\
\hline \multirow[t]{2}{*}{ Nasal swab } & PCR for respiratory viruses & & & & & & & $\mathrm{R}^{\mathrm{b}}$ & $R^{b}$ & & \\
\hline & PCR for Streptococcus carriage density & & & & & & & $R^{b}$ & $R^{b}$ & & \\
\hline
\end{tabular}

FGF fibroblast growth factor, IGF insulin-like growth factor, IGFBP insulin-like growth factor-binding protein, IL interleukin; $P T H r P$ parathyroid hormone-related protein, $R$ routine, $S$ subset, TNF tumor necrosis factor

${ }^{a}$ Routine analyses to be performed in all specimens collected; subset analyses to be performed in a sub-set of stored specimen aliquots

${ }^{\mathrm{b}} \mathrm{Nasal}$ swabbing is performed at any time from $1 \mathrm{wk}$ to $6 \mathrm{mo}$ of age if criteria for acute respiratory infection are met

USA) and maintained at less than $-70{ }^{\circ} \mathrm{C}$. At the completion of each workday, frozen aliquots are transported in the portable freezer or a liquid nitrogen canister to an upright $-80{ }^{\circ} \mathrm{C}$ freezer maintained with backup generator support and constant monitoring. One serum aliquot from each maternal and infant 3- and 6-month blood specimen is reserved at $2-8{ }^{\circ} \mathrm{C}$ for same- or next-day serum calcium measurement.

\section{Cord blood collection}

The technique and timing of umbilical cord clamping and cutting is determined by the attending physician or birthing attendant (i.e., cord clamping time is not set by the study protocol, but is recorded). As soon as possible within 30 minutes of delivery of the placenta, a site on the umbilical cord attached to the placenta is cleansed using dry cotton gauze to wipe away any maternal blood. The umbilical vein is cannulated, and blood is collected into collection tubes in the following order: (1) serum (red top) tube, (2) EDTA (lavender top) tube, and (3) trace element (blue top) tube with EDTA. Any remaining extractable blood is collected in EDTA (lavender top) tubes. Attempts are made to collect a total of 20-40 ml of cord blood. If feasible, the umbilical artery is cannulated and a serum (red top) tube also filled.

\section{Placental weight and specimen collection}

After collection of cord blood, the umbilical cord and membranes are removed from the placenta; the blood is drained; and the placenta is weighed to the nearest $0.5 \mathrm{~g}$ (iBALANCE i2500; My Weigh Canada, Vancouver, BC, Canada). Placental specimens from at least two quadrants of the placenta are collected and divided into three specimen types: (1) full disc biopsies of approximately $0.5-\mathrm{cm}$ thickness that are stored in $10 \%$ formalin at room temperature for histopathological examination; (2) small tissue samples (approximately $0.2 \mathrm{~cm}^{3}$ ) from the fetal surface of the placenta that are stored in RNA Save (Biological Industries Israel Beit Haemek, Kibbutz Beit Haemek, Israel) at $2-8{ }^{\circ} \mathrm{C}$ for $48 \mathrm{~h}$ and then transferred to $-80{ }^{\circ} \mathrm{C}$ until epigenetic and/or gene expression studies are done; and (3) small tissue samples (about 0.2 
$\mathrm{cm}^{3}$ ) from the maternal surface of the placenta that are stored and used similarly to the specimens from the fetal surface. Specimens from each quadrant are collected and pooled to create aliquots that represent the entire placental disc (one pooled histopathology sample and multiple pooled RNA Save samples from each of the fetal and maternal sides).

\section{Urine and breast milk specimen collection}

Maternal urine samples are self-collected into sterile plastic containers at baseline after enrollment (but before first supplement dose), during labor before delivery, and at the 6-month postpartum visit. For each sample, $1.5-\mathrm{ml}$ aliquots are stored at $-80{ }^{\circ} \mathrm{C}$ for future analyses. In addition, an aliquot from the intrapartum sample is held at $2-8{ }^{\circ} \mathrm{C}$ for same- or next-day analysis of the calcium:creatinine $(\mathrm{Ca}: \mathrm{Cr}$ ) ratio (see below). Infant urine samples are collected at the 6-month postnatal visit using infant urine collection bags that adhere to the perineum; $1.5-\mathrm{ml}$ aliquots are stored at $-80{ }^{\circ} \mathrm{C}$ for future analyses. At 3 and 6 months postpartum, hand-expressed midfeed breast milk samples are collected and stored at $-80{ }^{\circ} \mathrm{C}$ for future analyses. Between birth and 6 months of age, midturbinate nasal swabs for viral PCR will be collected from infants who meet clinical criteria for upper or lower respiratory tract infections. (Detailed methods for the ARI substudy will be presented elsewhere.)

\section{Laboratory analyses}

Vitamin D status is based on the serum 25(OH)D concentration [52] assessed by high-performance liquid chromatography tandem mass spectrometry performed at the Analytical Facility for Bioactive Molecules (Hospital for Sick Children, Toronto, ON, Canada). This assay captures $25(\mathrm{OH}) \mathrm{D}_{2}$ and $25(\mathrm{OH}) \mathrm{D}_{3}$, and enables separate quantification of the C3-epi-25(OH)D moiety. The current approach uses an Agilent 1290 Infinity high-performance liquid chromatography system (Agilent Technologies, Mississauga, ON, Canada) interfaced with an AB Sciex QTRAP 5500 mass spectrometer (SCIEX, Concord, ON, Canada), and separation by a Kinetex $2.6-\mu \mathrm{m}$ pentafluorophenyl $100 \mathrm{~mm} \times 2.1 \mathrm{~mm}$ column (Phenomenex, Torrance, CA, USA); however, iterative improvements to the assay system will ensure it remains state-of-the-art throughout the trial. In current use, average intraassay percent coefficient of variation $(\mathrm{CV} \%)$ is $4 \%$ and average interassay $\mathrm{CV} \%$ is $6 \%$. External quality control mechanisms include participation in the Vitamin D External Quality Assessment Scheme (DEQAS, http://www.deqas.org/; London, UK) and use of the National Institute of Standards and Technology (Gaithersburg, MD, USA) standard reference material.

Serum calcium is measured by colorimetric measurement of calcium-Arsenazo complex (Olympus-Beckman
Coulter autoanalyzer system; Beckman Coulter, Brea, CA, USA) in the clinical biochemistry laboratory at ICDDR,B (Dhaka, Bangladesh). Maternal and infant serum calcium is routinely monitored in all participants throughout the intervention phase of the trial (Table 3). To address specific mechanistic hypotheses, several other biochemical analytes will be measured in serum or plasma aliquots and urine samples from a subset of participants (Table 3). Details of these substudies will be described elsewhere. For epigenetic studies, DNA and mRNA will be extracted from blood and placental specimens using techniques previously described [53]. Genomic DNA will be subjected to bisulfite modification, and amplified PCR products will be analyzed by quantitative pyrosequencing to assess DNA methylation at several consecutive CpG sites within an approximately 150-bp region at the promoters of two genes involved in vitamin D metabolism (CYP27B1 and CYP24A1). Where possible, paternal whole blood specimens will be collected to enable the identification of allele-specific patterns. Gene expression (mRNA) from placental specimens (fetal tissue) will be assessed by real-time PCR. Epigenetic analyses will be performed at the Weksberg laboratory at the Hospital for Sick Children (Toronto, $\mathrm{ON}$, Canada). Molecular microbiological analyses of nasal specimens will be performed at the virology and microbiology laboratories at ICDDR,B. (Details of the ARI substudy will be described elsewhere.)

\section{Data management}

A site supervisor reviews all data collection forms for completeness and protocol deviations and/or violations before sending them to the data management center at ICDDR,B on a weekly basis. Electronic (scanned) versions of all forms are also regularly saved for long-term storage. The database was designed using SQL Server 2008 and data are entered using Visual Studio 2010 (both from Microsoft, Redmond, WA, USA). A set of range and consistency checks are built into the data capture system to provide immediate feedback to data entry personnel regarding errors or inconsistent data. Double data entry is used to further reduce the rate of date entry errors.

\section{Outcome measures}

The primary trial outcome measure is LAZ at 1 year of age, where length refers to an infant's fully extended crown-to-heel distance in the supine position. Growth faltering in resource-poor settings primarily occurs early [7], and any effects of the intervention are expected to be apparent by 1 year of age. Our preliminary trial data revealed a discernible effect of prenatal vitamin $D$ supplementation on infant LAZ at 1 year [26]. Because some infants might not be reached at exactly 1 year of 
age (52 weeks), measurements taken up to 60 weeks will be included in the primary 1-year outcome analysis, implying a range of 364-420 days. LAZ based on WHO growth standards will be used to account for sex imbalances between groups and variance in exact age at the time of measurement. Infant follow-up will continue to 2 years of age (with continued treatment masking) to establish the persistence of effects, to capture potential catch-up growth, and because stunting prevalence does not stabilize until 18-24 months of age [7]. To improve uniformity with respect to the timing of the 2-year visit, it will be scheduled at 24 months of age (104 weeks) but may be completed up to 27 months of age.

LAZs and z-scores for weight for age (WAZ), weight for length (WFLZ), and head circumference for age (HCAZ) will be calculated according to the sex-specific WHO growth standards (WHO-GS) [54, 55] using the STATA igrowup package (http://www.who.int/childgrowth/software/en/). Extreme z-scores will be flagged by the WHO Anthro software (less than -6 SD or greater than 6 SD for LAZ, greater than 5 or less than -6 for WAZ, greater than 5 or less than -5 for HCAZ, and greater than -5 or less than 5 for WFLZ). These extreme values will be manually reviewed to ensure they are not the result of data recording or entry errors. Real values that are extreme low outlying $\mathrm{z}$-scores are expected to be contributed primarily by infants who had early preterm births and of very low birth weight. Other secondary growth outcomes (UAL, MUAC, and RKL) and postnatal growth velocity will be analyzed using raw values or ageand sex-standardized z-scores based on WHO standards (except for RKL).

\section{Statistical analysis}

For the primary trial efficacy outcome analysis, mean LAZ (at 1 year of age) will be compared across groups using analysis of variance. To assess the effect of prenatal vitamin $\mathrm{D}$ on mean LAZ at 1 year of age, we plan to perform five primary between-group analyses: each prenatal vitamin $\mathrm{D}$ dose versus placebo (three pairwise comparisons), as well as comparisons between all adjacent vitamin D doses (two pairwise comparisons). Because the primary hypotheses relate to differences between two groups, each analysis will be akin to an independent-samples $t$ test. The primary effect measure for each comparison will be expressed as a mean difference between groups with $95 \%$ CI. However, an overall $\alpha$ for statistical significance for all five comparisons will be 0.05 (two-sided), and the Holm test will be used to account for multiple testing. Therefore, even if the $95 \%$ CI for a particular mean difference does not include zero, it is possible that it may not be considered statistically significant when all five comparisons are presented together. The postpartum effect analysis will be the comparison of mean LAZ at 1 year of age between infants of mothers who received 28,000 IU/wk versus placebo in the postpartum period (among women who received 28,000 IU/wk prenatally), using a statistical approach similar to that described for the prenatal effect analysis. The nominal $\alpha$ for statistical significance will be 0.05 (two-sided test) for this analysis. Because there is only one primary pairwise comparison related to the postpartum effect, no adjustment for the multiplicity of outcomes is planned.

In the primary analyses, an intention-to-treat (ITT) approach will be used, meaning participants will be considered exactly as randomized, regardless of supplementation adherence or completeness of follow-up and without adjustment for baseline covariates, and also without imputation for missing data; that is, infants for whom LAZ is unavailable at 1 year of age will be excluded from the analysis. However, because the trial is aimed at establishing a biological mechanism of effect, per-protocol analyses will also be performed by restricting to participants who consumed at least $90 \%$ of all scheduled doses. Conventional ITT analyses of postnatal growth outcomes typically disregard between-individual variations in GA at birth; however, because the WHOGS was based on a full-term cohort [54], this may lead to differential misclassification of preterm infants with respect to LAZ if length is plotted on the growth chart using the chronological age. Although we plan to follow the convention in the primary analysis, we will perform a sensitivity analysis in which LAZ will be assigned to preterm infants using the age corrected for GA at birth (rather than chronological age). Several other sensitivity analyses are planned. Multivariable adjustment will be used for covariates that substantially differ across groups at baseline. A missingness analysis will be performed to understand the pattern of missing data (in particular to detect differential loss of data across groups), and multiple imputation methods will be used in sensitivity analyses to correct for these losses. Subgroup and interaction analyses will be based on the following covariates: GA at birth, infant sex, maternal baseline vitamin $D$ status, maternal height, and maternal supplement adherence.

Two secondary approaches will be used to examine the effects of vitamin D on linear growth. Regression spline models, with knots at the major scheduled follow-up visit time points, will be used to analyze changes in mean LAZ over discrete time intervals. Interaction terms for time and group allocation will be used to test between-group differences in the changes in LAZ during discrete time intervals. As well, participants will be classified as "stunted" if LAZ is less than -2 , and both cross-sectional and longitudinal comparisons will be undertaken to assess the relative risk of stunting across groups. In longitudinal analyses of LAZ or 
stunting described above, generalized estimating equations with robust variance estimation will be employed to account for within-subject correlation of repeated measures [56].

Summary measures (e.g., means, frequencies, proportions, incidence rates) and effect estimates (i.e., regression coefficients) will be reported as point estimates and $95 \%$ confidence intervals. In general, risk ratios with corresponding CIs will be used to compare dichotomous outcomes across trial groups (e.g., prevalence of SGA), and differences in means will be used for between-group comparisons of continuous variables. $P$ values will be reported to three decimal places, with $P$ values less than 0.001 reported as less than 0.001 . Statistical analyses will be performed using the STATA software package (StataCorp, College Station, TX, USA) with analyses of primary trial outcomes performed in a blinded fashion. Plans of analysis to address secondary objectives will be presented elsewhere.

\section{Participant safety monitoring}

Participant safety during the intervention phase is monitored by study personnel based on (1) a checklist of maternal symptoms that may indicate vitamin D toxicity or other medical concerns during weekly followup visits, (2) passive and active monitoring for maternal and infant clinical events, and (3) maternal serum calcium measurement at scheduled intervals during the intervention phase (baseline, 30 weeks of gestation, delivery, 3 months postpartum, and 6 months postpartum). Secondary safety measures include infant serum calcium measurement at 3 and 6 months of age and maternal urinary calcium:creatinine at the time of delivery.

Serum calcium is the best available biomarker of vitamin D toxicity $[57,58]$; therefore, hypercalcemia is the primary biochemical safety outcome. Nonetheless, on the basis of our own experience with doses equivalent to up to $5000 \mathrm{IU} /$ day (see above) and previously published trials conducted in the United States using doses up to $4000 \mathrm{IU} /$ day during pregnancy [43] and up to $6400 \mathrm{IU} /$ day during lactation [59], we do not anticipate any episodes of true hypercalcemia or other supplement-related adverse events.

Possible hypercalcemia is defined as a single serum calcium concentration above $2.60 \mathrm{mmol} / \mathrm{L}$. This threshold is based on a conservative upper limit of the normal range that accommodates expected variation in serum calcium throughout pregnancy [60], but it avoids the unnecessary operational complexity of using a normal range that varies by stage of gestation. Any participant with possible hypercalcemia detected through scheduled blood sampling (or at the time of a clinical serious adverse event; e.g., hospitalization) will be asked to provide a second sample within $24 \mathrm{~h}$ of the time that the study physician receives a report of an abnormal value. Abnormal serum calcium results will always be reported and confirmed before the time of the following week's supplement dose (to enable a decision to be made about withholding supplementation). Severe derangements in serum calcium will be managed as urgently as possible, including referral for hospitalization.

Confirmed hypercalcemia is defined as serum calcium concentration above $2.60 \mathrm{mmol} / \mathrm{L}$ in two consecutive specimens and will be considered the primary supplement-related adverse event. The second, confirmatory specimen is required owing to the possibility of a laboratory error, but clinical management will not await the second assay if the participant is symptomatic. If the repeat serum calcium is normal, then supplementation will continue and a further repeat serum calcium will be measured 1 week after the first abnormal result to confirm that it remains within the normal range. In the unlikely event that a participant has confirmed hypercalcemia, study personnel will follow the clinical course until normalization of serum calcium or 1 month postpartum (whichever occurs later). Participants with mild and asymptomatic hypercalcemia may not require referral or treatment beyond the cessation of supplementation and will continue to participate in study follow-up. Participants with confirmed or suspected moderate to severe hypercalcemia (serum $\mathrm{Ca}>2.80$ $\mathrm{mmol} / \mathrm{L}$ and/or symptomatic) will be assessed at a hospital by a clinician with expertise in the treatment of hypercalcemia.

In previous prenatal vitamin $\mathrm{D}$ supplementation trials in Dhaka, we used regular monitoring of maternal urinary $\mathrm{Ca}: \mathrm{Cr}$ ratio as a screening measure for vitamin $\mathrm{D}$ toxicity $[16,40]$. However, this test is non-specific and frequently led to the need for repeat testing that was burdensome but uninformative; in no cases did an isolated $\mathrm{Ca}: \mathrm{Cr}$ ratio indicate vitamin $\mathrm{D}$ toxicity. Therefore, regular urine $\mathrm{Ca}: \mathrm{Cr}$ ratio assessment is not employed as a primary clinical safety monitoring tool in the present trial. However, we will measure urinary $\mathrm{Ca}: \mathrm{Cr}$ ratio in mothers at delivery as a screening test for hypercalciuria rather than for overt vitamin $\mathrm{D}$ toxicity. Abnormal values $(\mathrm{Ca}: \mathrm{Cr}$ ratio $>1 \mathrm{mmol} / \mathrm{mmol}$ ) will prompt repeat testing, and either of the following criteria will be considered a presumptive diagnosis of hypercalciuria: two consecutive urine samples with $\mathrm{Ca} \mathrm{Cr}$ ratio above 1 $\mathrm{mmol} / \mathrm{mmol}$ or one urine sample with $\mathrm{Ca}: \mathrm{Cr}$ ratio above $1 \mathrm{mmol} / \mathrm{mmol}$ in the presence of persistent symptoms suggestive of possible uro- and/or nephrolithiasis. Participants with persistent symptoms of renal colic or hypercalciuria will be referred for renal ultrasound to assess for the presence of urolithiasis or nephrolithiasis. Participants with uro- or nephrolithiasis will be referred for 
consideration by the DSMB for a decision regarding unblinding and possible discontinuation of the study supplement, which is to be decided on a case-by-case basis. Biochemical evidence of hypercalciuria alone will not trigger urgent DSMB review, but it will be reviewed at regular intervals. However, participants with hypercalciuria and an absence of stones will undergo repeat urine $\mathrm{Ca} \mathrm{Cr}$ ratio assessment 1 month after initial diagnosis. If hypercalciuria is persistent, a repeat ultrasound will be undertaken.

When caregivers permit scheduled infant blood sampling, serum calcium will be routinely measured. The normal range of infant serum calcium is higher than in adults, and normal values may be as high as $3.05 \mathrm{mmol} / \mathrm{L}$ [61-66]. However, because there are few reference data for infants specifically at 3 and 6 months, we have chosen to use a more conservative threshold of greater than 2.80 $\mathrm{mmol} / \mathrm{L}$ to define above-range infant values at the 3- and 6-month visits, which will prompt repeat assessment.

Clinical events will be monitored and documented by study physicians, and serious adverse events will include all deaths and hospitalizations (with the exception of admissions to the hospital for uncomplicated vaginal or cesarean delivery). The only clinical events that will routinely lead to discontinuation of study supplementation are those adverse events that are ascertained to be supplement-related or the diagnosis of a medical condition that potentially or theoretically increases sensitivity to vitamin D supplementation following clinical review by the Trial Steering Committee (TSC): confirmed hypercalcemia (see definition above), symptomatic vitamin D deficiency diagnosed by a study physician or consultant physician (e.g., osteomalacia), fetal or infant death, or onset of a medical condition or initiation of a medication following enrollment that may reasonably predispose to vitamin D sensitivity, altered vitamin D metabolism, and/or hypercalcemia (e.g., tuberculosis or therapy for tuberculosis, sarcoidosis, renal and/or ureteral stones, parathyroid disease, renal or liver failure, or use of anticonvulsants). The occurrence of a serious adverse event, in of itself, will not automatically lead to study supplementation cessation.

Research ethics committee/institutional review board approval was obtained from the Hospital for Sick Children Research Ethics Board (REB1000039072) and the ICDDR,B Ethical Review Committee (ERC; PR number 13055). A DSMB was created by the ERC of ICDDR,B. An external international member of the TSC reviews reports submitted to the DSMB and reports to the TSC. There are no formal interim analyses of efficacy or stopping rules planned for this trial.

\section{Discussion}

Since the publication of the 2010 IOM report on vitamin $\mathrm{D}$ and the design of the MDIG trial and dosing regimens in 2012, there have been several new reports of biochemical and clinical findings of prenatal vitamin D supplementation trials conducted in Iran [67-69], the United Arab Emirates [70], Pakistan [71], New Zealand [72], and Australia [73]. However, in none of these studies did researchers report postnatal infant growth outcomes, and all trials enrolled fewer than 100 participants per arm, suggesting they lacked power to detect effects on most pregnancy-related and infant clinical outcomes. The results of a relatively larger trial $(N=549)$ recently completed in rural Pakistan (NCT01229189) have not yet been released publicly. There are at least three other large trials underway: the Maternal Vitamin D Osteoporosis Study, for which the primary outcome is neonatal whole body bone mineral content [74]; the Vitamin D Antenatal Asthma Reduction Trial [75]; and the Vitamin D Supplementation during Pregnancy for Prevention of Asthma in Childhood (ABCvitaminD trial; ClinicalTrials.gov identifier NCT00856947). However, these and other registered prenatal vitamin D trials are being conducted in high-income countries.

The MDIG trial will therefore offer unique insights into the effect of improving maternal-infant vitamin D status in a low-income setting where vitamin D insufficiency is common and there is a high prevalence of infant linear growth faltering. The trial is primarily designed and powered to evaluate the dose-response effects of vitamin D on infant length, an outcome that is directly aligned with the classical role of vitamin $\mathrm{D}$ in mineral metabolism and bone health but may also be interpreted as a surrogate marker for organ (including brain) growth and development. Serial specimen collection and frequent clinical follow-up will enable a range of secondary objectives to be addressed, including tests of mechanistic hypotheses (e.g., mediation of the beneficial vitamin $\mathrm{D}$ supplement effect on skeletal length by PTH suppression) and assessments of the effects of the intervention on infant morbidity (in particular, the incidence of acute respiratory infections in the first 6 months after birth). Longer-term surveillance of the infant cohort will ideally enable the assessment of other important clinical outcomes (e.g., asthma, neurodevelopment) that may be influenced by the non-classical role of vitamin D in immunoregulation and extraskeletal cellular proliferation and differentiation.

\section{Trial status}

As of July 2015, enrollment is ongoing.

\section{Additional files}

Additional file 1: Summary of the rationale for the maternal prenatal and postpartum vitamin D3 doses selected for the trial. 
Additional file 2: Overview of study activities scheduled for each participant enrolled in the trial.

\section{Abbreviations}

ARI: acute respiratory infection; $\mathrm{Ca}: \mathrm{Cr}$ : calcium-creatinine ratio; $\mathrm{Cl}$ : confidence interval; DSMB: Data and Safety Monitoring Board;

EDTA: ethylenediaminetetraacetic acid; ERC: Ethical Review Committee; FGF: fibroblast growth factor; GA: gestational age; Hb: hemoglobin; $\mathrm{HC}$ : head circumference; HCAZ: head circumference for age z-score;

ICDDR: International Centre for Diarrhoeal Disease Research; IGF: insulin-like growth factor; IGFBP: insulin-like growth factor-binding protein;

IL: interleukin; IOM: Institute of Medicine; ITT: intention to treat; 25(OH)D: 25 hydroxyvitamin D; RKL: rump-to-knee length; LAZ: length for age z-score; LMP: last menstrual period; MCHTI: Maternal and Child Health Training Institute; MDIG: Maternal Vitamin D for Infant Growth; MUAC: mid-upper arm circumference; POC: point of care; PTH: parathyroid hormone; PTHrP: parathyroid hormone-related protein; SGA: small for gestational age; TIPT: Toronto Institute of Pharmaceutical Technology; TNF: tumor necrosis factor; TSC: trial steering committee; UAL: upper arm length; WAZ: Weight for age z-score; WFLZ: weight for length z-score; WHO: World Health Organization; WHO-GS: World Health Organization growth standards.

\section{Competing interests}

The authors declare that they have no competing interests.

\section{Authors' contributions}

DER originated the idea for the study, led the protocol design, and wrote the manuscript. ADG, SKM, MMI, SHZ, TA, PSS, KEM, RW, SC, and RS participated in the design of the study. BP and MCD were involved in the development of the study protocol. SSS was involved in the development of the study protocol and its implementation. ARW (trial statistician) was involved in study design and the data analysis plan. AAM participated in the design of the study and leads the project's implementation in Bangladesh. All authors read and approved the final manuscript.

\section{Acknowledgments}

We thank the following individuals for their contributions to the design or development of the study procedures and/or trial implementation: Eszter Papp, Lisa Pell, Ashley Motran, Thulasi Thiruchselvam, Jo-Anna Baxter, Hayley Craig-Barnes, Michael Leadley, Pilar Zanoni, Farhana Keya, Tahmid Kashem Qazi, Sadeq-ur Rahman, Kazi Taib Mamun, Frank Martinuzzi. We also thank Dr. Ishrat Jahan, Director of MCHTI, and Kazi Moksedur Rahman, Executive Director of Shimantik (Bangladesh).

MDIG Trial Steering Committee (TSC): Tahmeed Ahmed, Alison Gernand, David Hamer (external member), M. Munirul Islam, Abdullah Al Mahmud (site principle investigator [PE]), Shaun Morris, Kellie Murphy, Daniel Roth (PI), Rashed Shah, Prakesh Shah, Rosanna Weksberg, Andrew Willan, and Stanley Zlotkin. The trial is funded by the Bill and Melinda Gates Foundation (BMGF, OPP1066764). The funder was not involved in the writing of the manuscript or the decision to submit the manuscript for publication. ADG's research is supported by the Eunice Kennedy Shriver National Institute of Child Health and Human Development of the National Institutes of Health $(\mathrm{NIH})$ under BIRCWH award number K12HD055882, Career Development Program in Women's Health Research at Penn State. The content of this article is solely the responsibility of the authors and does not necessarily represent the official views of the $\mathrm{NIH}$.

\section{Author details}

'Department of Paediatrics, University of Toronto and the Centre for Global Child Health, Hospital for Sick Children, 686 Bay Street, Toronto, ON, Canada. ${ }^{2}$ Child Health Evaluative Sciences, SickKids Research Institute, Hospital for Sick Children, 686 Bay Street, Toronto, ON, Canada. ${ }^{3}$ Department of Nutritional Sciences, Penn State University, 110 Chandlee Laboratory, University Park, PA, USA. ${ }^{4}$ Centre for Nutrition and Food Security, International Centre for Diarrhoeal Disease Research (ICDDR,B), 68 Shaheed Tajuddin Ahmed Sarani, Mohakhali, Dhaka 1212, Bangladesh. ${ }^{5}$ Division of Neonatology, Mt. Sinai Hospital, 600 University Avenue, Toronto, ON, Canada. ${ }^{6}$ Department of Obstetrics and Gynecology, University of Toronto and Mt. Sinai Hospital, 600 University Avenue, Toronto, ON, Canada. ${ }^{7}$ Genetics and Genome Biology, SickKids Research Institute, Hospital for Sick Children, 686 Bay Street, Toronto,
ON, Canada. ${ }^{8}$ Department of Health and Nutrition, Save the Children USA, 2000 L Street NW, Suite 500, Washington, DC, USA. ${ }^{9}$ Centre for Child and Adolescent Health, International Centre for Diarrhoeal Disease Research (ICDDR,B), 68 Shaheed Tajuddin Ahmed Sarani, Mohakhali, Dhaka 1212, Bangladesh.

Received: 12 March 2015 Accepted: 26 June 2015

Published online: 14 July 2015

\section{References}

1. Liu L, Oza S, Hogan D, Perin J, Rudan I, Lawn JE, et al. Global, regional, and national causes of child mortality in 2000-13, with projections to inform post-2015 priorities: an updated systematic analysis. Lancet. 2015;385(9966):430-40

2. Black RE, Allen LH, Bhutta ZA, Caulfield LE, de Onis M, Ezzati M, et al. Maternal and child undernutrition: global and regional exposures and health consequences. Lancet. 2008:371(9608):243-60.

3. Stevens GA, Finucane MM, Paciorek CJ, Flaxman SR, White RA, Donner AJ, et al. Trends in mild, moderate, and severe stunting and underweight, and progress towards MDG 1 in 141 developing countries: a systematic analysis of population representative data. Lancet. 2012;380(9844):824-34.

4. National Institute of Population Research and Training (NIPORT), Mitra and Associates, ICF International. Bangladesh demographic and health survey 2011. Dhaka, Bangladesh/Calverton, MD: NIPORT, Mitra and Associates, ICF International; January 2013. http://dhsprogram.com/pubs/pdf/FR265/ FR265.pdf. Accessed 5 July 2015.

5. Victora CG, Adair L, Fall C, Hallal PC, Martorell R, Richter $L$, et al. Maternal and child undernutrition: consequences for adult health and human capital. Lancet. 2008;371(9609):340-57.

6. Black RE, Victora CG, Walker SP, Bhutta ZA, Christian P, de Onis M, et al Maternal and child undernutrition and overweight in low-income and middle-income countries. Lancet. 2013;382(9890):427-51.

7. Victora CG, de Onis M, Hallal PC, Blössner M, Shrimpton R. Worldwide timing of growth faltering: revisiting implications for interventions. Pediatrics. 2010;125(3):e473-80.

8. Prentice AM, Moore SE, Fulford AJ. Growth faltering in low-income countries. World Rev Nutr Diet. 2013:106:90-9.

9. Piwoz E, Sundberg S, Rooke J. Promoting healthy growth: what are the priorities for research and action? Adv Nutr. 2012;3(2):234-41.

10. Bikle DD. Vitamin D, metabolism, mechanism of action, and clinical applications. Chem Biol. 2014;21(3):319-29.

11. Soliman AT, Al Khalaf F, Alhemaidi N, Al Ali M, Al Zyoud M, Yakoot K. Linear growth in relation to the circulating concentrations of insulin-like growth factor I, parathyroid hormone, and 25-hydroxy vitamin D in children with nutritional rickets before and after treatment: endocrine adaptation to vitamin D deficiency. Metabolism. 2008;57(1):95-102.

12. Rajah J, Jubeh JA, Haq A, Shalash A, Parsons H. Nutritional rickets and z scores for height in the United Arab Emirates: to D or not to D? Pediatr Int. 2008;50(4):424-8

13. Bereket A, Cesur Y, Özkan B, Adal E, Turan S, Onan SH, et al. Circulating insulin-like growth factor binding protein-4 (IGFBP-4) is not regulated by parathyroid hormone and vitamin $D$ in vivo: evidence from children with rickets. J Clin Res Pediatr Endocrinol. 2010;2(1):17-20.

14. Kumar GT, Sachdev HS, Chellani H, Rehman AM, Singh V, Arora H, et al. Effect of weekly vitamin D supplements on mortality, morbidity, and growth of low birthweight term infants in India up to age 6 months: randomised controlled trial. BMJ. 2011;342:d2975.

15. Palacios C, Gonzalez L. Is vitamin D deficiency a major global public health problem? J Steroid Biochem Mol Biol. 2014;144 Pt A:138-45.

16. Roth DE, Al Mahmud A, Raqib R, Akhtar E, Perumal N, Pezzack B, et al. Randomized placebo-controlled trial of high-dose prenatal third-trimester vitamin D3 supplementation in Bangladesh: the AViDD trial. Nutr J. 2013;12:47.

17. Ross AC, Manson JE, Abrams SA, Aloia JF, Brannon PM, Clinton SK, et al. The 2011 report on dietary reference intakes for calcium and vitamin D from the Institute of Medicine: what clinicians need to know. J Clin Endocrinol Metab. 2011:96(1):53-8

18. Salle BL, Delvin EE, Lapillonne A, Bishop NJ, Glorieux FH. Perinatal metabolism of vitamin D. Am J Clin Nutr. 2000;71(5 Suppl):1317S-24S.

19. Hanieh S, Ha TT, De Livera AM, Simpson JA, Thuy TT, Khuong NC, et al. Antenatal and early infant predictors of postnatal growth in rural Vietnam: a prospective cohort study. Arch Dis Child. 2015;100(2):165-73. 
20. Leffelaar ER, Vrijkotte TGM, van Eijsden M. Maternal early pregnancy vitamin $D$ status in relation to fetal and neonatal growth: results of the multi-ethnic Amsterdam Born Children and their Development cohort. Br J Nutr. 2010;104(1):108-17.

21. Eckhardt $C L$, Gernand $A D$, Roth $D E$, Bodnar $L M$ : Maternal vitamin D status and infant anthropometry in a US multi-centre cohort study. Ann Hum Biol. In press. doi:10.3109/03014460.2014.954616.

22. Prentice A, Jarjou LM, Goldberg GR, Bennett J, Cole TJ, Schoenmakers I. Maternal plasma 25-hydroxyvitamin D concentration and birthweight, growth and bone mineral accretion of Gambian infants. Acta Paediatr. 2009;98(8):1360-2.

23. Specker BL. Does vitamin D during pregnancy impact offspring growth and bone? Proc Nutr Soc. 2012;71(1):38-45.

24. Brooke OG, Brown IR, Bone CD, Carter ND, Cleeve HJ, Maxwell JD, et al. Vitamin D supplements in pregnant Asian women: effects on calcium status and fetal growth. Br Med J. 1980;280(6216):751-4.

25. Brooke OG, Butters F, Wood C. Intrauterine vitamin D nutrition and postnatal growth in Asian infants. Br Med J (Clin Res Ed). 1981;283(6298):1024.

26. Roth DE, Perumal N, Al Mahmud A, Baqui AH. Maternal vitamin D3 supplementation during the third trimester of pregnancy: effects on infant growth in a longitudinal follow-up study in Bangladesh. J Pediatr. 2013;163(6):1605-11.e3.

27. Finch SL, Rauch F, Weiler HA. Postnatal vitamin D supplementation following maternal dietary vitamin $D$ deficiency does not affect bone mass in weanling guinea pigs. J Nutr. 2010;140(9):1574-81.

28. Gernand AD, Simhan HN, Klebanoff MA, Bodnar LM. Maternal serum 25hydroxyvitamin $D$ and measures of newborn and placental weight in a U.S. multicenter cohort study. J Clin Endocrinol Metab. 2013;98(1):398-404.

29. Thorne-Lyman A, Fawzi WW. Vitamin D during pregnancy and maternal, neonatal and infant health outcomes: a systematic review and metaanalysis. Paediatr Perinat Epidemiol. 2012;26 Suppl 1:75-90.

30. Aghajafari F, Nagulesapillai T, Ronksley PE, Tough SC, O'Beirne M, Rabi DM. Association between maternal serum 25-hydroxyvitamin D level and pregnancy and neonatal outcomes: systematic review and meta-analysis of observational studies. BMJ. 2013;346:f1169.

31. Harvey NC, Holroyd C, Ntani G, Javaid K, Cooper P, Moon R, et al. Vitamin D supplementation in pregnancy: a systematic review. Health Technol Assess. 2014;18(45):1-190. doi:10.3310/hta18450.

32. De-Regil LM, Palacios C, Ansary A, Kulier R, Peña-Rosas JP. Vitamin D supplementation for women during pregnancy. Cochrane Database Syst Rev. 2012;2:CD008873.

33. World Health Organization. Guideline: vitamin D supplementation in pregnant women. Geneva: World Health Organization; 2012. http:// apps.who.int/iris/bitstream/10665/85313/1/9789241504935_eng.pdf. Accessed 5 July 2015.

34. Chan AW, Tetzlaff JM, Gøtzsche PC, Altman DG, Mann H, Berlin JA, et al. SPIRIT 2013 explanation and elaboration: guidance for protocols of clinical trials. BMJ. 2013;346:e7586.

35. Centre for Urban Studies, National Institute of Population Research and Training, and MEASURE Evaluation. Slums of Urban Bangladesh: Mapping and Census, 2005. Dhaka, Bangladesh and Chapel Hill, USA: Centre for Urban Studies, National Institute of Population Research and Training, and MEASURE Evaluation; 2006. http://www.cpc.unc.edu/measure/publications/ tr-06-35/at_download/document. Accessed 11 July 2015

36. Clinical Practice Obstetrics Committee, Maternal Fetal Medicine Committee, Delaney M, Roggensack A, Leduc DC, Ballermann C, et al. Guidelines for the management of pregnancy at $41+0$ to $42+0$ weeks. J Obstet Gynaecol Can. 2008;30(9):800-23.

37. Schulz KF, Grimes DA. Generation of allocation sequences in randomised trials: chance, not choice. Lancet. 2002;359(9305):515-9.

38. Ross AC, Taylor CL, Yaktine AL, Del Valle HB, editors. Committee to Review Dietary Reference Intakes for Vitamin D and Calcium, Institute of Medicine. Dietary reference intakes: calcium and vitamin D. Washington, DC: The National Academies Press; 2011.

39. Ish-Shalom S, Segal E, Salganik T, Raz B, Bromberg IL, Vieth R. Comparison of daily, weekly, and monthly vitamin D3 in ethanol dosing protocols for two months in elderly hip fracture patients. J Clin Endocrinol Metab. 2008;93(9):3430-5.

40. Roth DE, Al Mahmud A, Raqib R, Akhtar E, Black RE, Baqui AH. Pharmacokinetics of high-dose weekly oral vitamin D3 supplementation during the third trimester of pregnancy in Dhaka, Bangladesh. Nutrients. 2013;5(3):788-810.
41. Heaney RP, Davies KM, Chen TC, Holick MF, Barger-Lux MJ. Human serum 25hydroxycholecalciferol response to extended oral dosing with cholecalciferol. Am J Clin Nutr. 2003;77(1):204-10.

42. Aloia JF, Patel M, Dimaano R, Li-Ng M, Talwar SA, Mikhail M, et al. Vitamin D intake to attain a desired serum 25 -hydroxyvitamin D concentration. Am J Clin Nutr. 2008;87(6):1952-8.

43. Hollis BW, Johnson D, Hulsey TC, Ebeling M, Wagner CL. Vitamin D supplementation during pregnancy: double blind, randomized clinical trial of safety and effectiveness. J Bone Miner Res. 2011;26(10):2341-57.

44. Wagner D, Sidhom G, Whiting SJ, Rousseau D, Vieth R. The bioavailability of vitamin $\mathrm{D}$ from fortified cheeses and supplements is equivalent in adults. J Nutr. 2008;138(7):1365-71.

45. World Health Organization. Guideline: calcium supplementation in pregnant women. Geneva: World Health Organization; 2013. http:// apps.who.int/iris/bitstream/10665/85120/1/9789241505376_eng.pdf. Accessed 5 July 2015.

46. Cheikh Ismail L, Knight HE, Bhutta Z, Chumlea WC, International Fetal and Newborn Growth Consortium for the 21st Century (INTERGROWTH-21st). Anthropometric protocols for the construction of new international fetal and newborn growth standards: the INTERGROWTH-21st Project. BJOG. 2013;120 Suppl 2:42-7.

47. Centers for Disease Control and Prevention. National Health and Nutrition Examination Survey: anthropometry procedures manual. Hyattsville, MD: National Center for Health Statistics, Centers for Disease Control and Prevention; January 2009. http://www.cdc.gov/nchs/data/nhanes/ nhanes_09_10/BodyMeasures_09.pdf. Accessed 5 July 2015.

48. National Household Survey Capability Programme. How to weigh and measure children: assessing the nutritional status of young children in household surveys. New York: Department of Technical Co-Operation for Development and Statistical Office, United Nations; 1986. http://unstats.un.org/ unsd/publication/unint/dp_un_int_81_041_6E.pdf. Accessed 5 July 2015.

49. Snyder RG, Schneider LW, Owings CL, Reynolds HM, Golomb DH, Schork MA. Anthropometry of infants, children, and youths to age 18 for product safety design. Final report (UM-HSRI-77-17). Ann Arbor, Ml: Highway Safety Research Institute, University of Michigan; 31 May 1977. http://ovrt.nist.gov/ projects/anthrokids/child77lnk.pdf. Accessed 5 July 2015.

50. de Onis M, Onyango AW, Van den Broeck J, Chumlea WC, Martorell R. Measurement and standardization protocols for anthropometry used in the construction of a new international growth reference. Food Nutr Bull. 2004;25(1 Suppl):S27-36.

51. Cheikh Ismail L, Knight HE, Ohuma EO, Hoch L, Chumlea WC. International Fetal and Newborn Growth Consortium for the 21st Century. Anthropometric standardisation and quality control protocols for the construction of new, international, fetal and newborn growth standards: the INTERGROWTH-21st Project. BJOG. 2013;120 Suppl 2:48-55.

52. Seamans KM, Cashman KD. Existing and potentially novel functional markers of vitamin D status: a systematic review. Am J Clin Nutr. 2009:89(6):1997S-2008S.

53. Choufani S, Shapiro JS, Susiarjo M, Butcher DT, Grafodatskaya D, Lou Y, et al. A novel approach identifies new differentially methylated regions (DMRs) associated with imprinted genes. Genome Res. 2011;21(3):465-76.

54. World Health Organization. WHO child growth standards: length/height-forage, weight-for-age, weight-for-length, weight-for-height and body mass index-for-age: methods and development. Geneva: World Health Organization; 2006. http://apps.who.int/iris/bitstream/10665/43413/1/ 924154693X_eng.pdf. Accessed 5 July 2015.

55. Department of Nutrition for Health and Development, World Health Organization. WHO child growth standards: head circumference-for-age, arm circumference-for-age, triceps skinfold-for-age and subscapular skinfoldfor-age: methods and development. Geneva: World Health Organization; 2007. http://www.who.int/childgrowth/standards/second_set/technical_ report_2.pdf?ua=1. Accessed 5 July 2015.

56. Zeger SL, Liang KY, Albert PS. Models for longitudinal data: a generalized estimating equation approach. Biometrics. 1988;44(4):1049-60.

57. Hathcock JN, Shao A, Vieth R, Heaney R. Risk assessment for vitamin D. Am J Clin Nutr. 2007;85(1):6-18.

58. Roth DE. Vitamin D, supplementation during pregnancy: safety considerations in the design and interpretation of clinical trials. J Perinatol. 2011;31(7):449-59.

59. Wagner $C L$, Hulsey TC, Fanning D, Ebeling M, Hollis BW. High-dose vitamin D3 supplementation in a cohort of breastfeeding mothers and their infants: a 6-month follow-up pilot study. Breastfeed Med. 2006;1(2):59-70. 
60. Larsson A, Palm M, Hansson LO, Axelsson O. Reference values for clinical chemistry tests during normal pregnancy. BJOG. 2008;115(7):874-81.

61. Ghoshal AK, Soldin SJ. Evaluation of the Dade Behring Dimension RxL: integrated chemistry system-pediatric reference ranges. Clin Chim Acta. 2003;331(1-2):135-46.

62. Chan MK, Seiden-Long I, Aytekin M, Quinn F, Ravalico T, Ambruster D, et al. Canadian Laboratory Initiative on Pediatric Reference Interval Database (CALIPER): pediatric reference intervals for an integrated clinical chemistry and immunoassay analyzer, Abbott ARCHITECT ci8200. Clin Biochem. 2009;42(9):885-91.

63. Kulasingam V, Jung BP, Blasutig IM, Baradaran S, Chan MK, Aytekin M, et al. Pediatric reference intervals for 28 chemistries and immunoassays on the Roche cobas 6000 analyzer - a CALIPER pilot study. Clin Biochem. 2010;43(13-14):1045-50.

64. Blasutig IM, Jung B, Kulasingam V, Baradaran S, Chen Y, Chan MK, et al. Analytical evaluation of the VITROS 5600 Integrated System in a pediatric setting and determination of pediatric reference intervals. Clin Biochem. 2010;43(13-14):1039-44.

65. Ridefelt $\mathrm{P}$, Aldrimer $\mathrm{M}$, Rödöö PO, Niklasson F, Jansson L, Gustafsson J, et al. Population-based pediatric reference intervals for general clinical chemistry analytes on the Abbott Architect ci8200 instrument. Clin Chem Lab Med. 2012;50(5):845-51.

66. Roizen JD, Shah V, Levine MA, Carlow DC. Determination of reference intervals for serum total calcium in the vitamin D-replete pediatric population. J Clin Endocrinol Metab. 2013;98(12):E1946-50.

67. Soheilykhah S, Mojibian M, Moghadam MJ, Shojaoddiny-Ardekani A. The effect of different doses of vitamin $D$ supplementation on insulin resistance during pregnancy. Gynecol Endocrinol. 2013;29(4):396-9.

68. Asemi Z, Samimi M, Tabassi Z, Shakeri H, Esmaillzadeh A. Vitamin D supplementation affects serum high-sensitivity C-reactive protein, insulin resistance, and biomarkers of oxidative stress in pregnant women. J Nutr. 2013;143(9):1432-8.

69. Shakiba M, Iranmanesh MR. Vitamin D requirement in pregnancy to prevent deficiency in neonates: a randomised trial. Singapore Med J. 2013;54(5):285-8.

70. Dawodu A, Saadi HF, Bekdache G, Javed Y, Altaye M, Hollis BW. Randomized controlled trial $(\mathrm{RCT})$ of vitamin $\mathrm{D}$ supplementation in pregnancy in a population with endemic vitamin D deficiency. J Clin Endocrinol Metab. 2013;98(6):2337-46.

71. Hossain N, Kanani FH, Ramzan S, Kausar R, Ayaz S, Khanani R, et al. Obstetric and neonatal outcomes of maternal vitamin $D$ supplementation: results of an open-label, randomized controlled trial of antenatal vitamin D supplementation in Pakistani women. J Clin Endocrinol Metab. 2014;99(7):2448-55.

72. Grant CC, Stewart AW, Scragg R, Milne T, Rowden J, Ekeroma A, et al. Vitamin D during pregnancy and infancy and infant serum 25hydroxyvitamin D concentration. Pediatrics. 2014;133(1):e143-153.

73. Yap C, Cheung NW, Gunton JE, Athayde N, Munns CF, Duke A, et al. Vitamin D supplementation and the effects on glucose metabolism during pregnancy: a randomized controlled trial. Diabetes Care. 2014;37(7):1837-44.

74. Harvey NC, Javaid K, Bishop N, Kennedy S, Papageorghiou AT, Fraser R, et al. MAVIDOS Maternal Vitamin D Osteoporosis Study: study protocol for a randomized controlled trial. The MAVIDOS Study Group. Trials. 2012;13:13.

75. Litonjua AA, Lange NE, Carey VJ, Brown S, Laranjo N, Harshfield BJ, et al. The Vitamin D Antenatal Asthma Reduction Trial (VDAART): rationale, design, and methods of a randomized, controlled trial of vitamin D supplementation in pregnancy for the primary prevention of asthma and allergies in children. Contemp Clin Trials. 2014:38(1):37-50.

\section{Submit your next manuscript to BioMed Central and take full advantage of:}

- Convenient online submission

- Thorough peer review

- No space constraints or color figure charges

- Immediate publication on acceptance

- Inclusion in PubMed, CAS, Scopus and Google Scholar

- Research which is freely available for redistribution 